\title{
Justification of Urgent Brain CT Examinations at Medium Size Hospital, Jerusalem
}

\author{
Anan Al-Tell', Majdal Hjouj ${ }^{2}$, Muntaser S. Ahmad ${ }^{3, *}$, and Hjouj Mohammad ${ }^{1,4}$
}

${ }^{1}$ Radiology and Medical imaging department, Maqassed Hospital, Jerusalem, Palestine; ${ }^{2}$ Medical School, Al-Quds University, Abu Deis - Main Campus, Jerusalem, Palestine; ${ }^{3}$ Department of Medical Physics and Radiation Science, School of Physics, University Sains Malaysia, 11800 Penang, Malaysia; ${ }^{4}$ Medical Imaging Department, Faculty of Health Professions, Al-Quds University, Jerusalem, Palestine.

Received: October 25, 2019 / Accepted: December 8, 2019

\begin{abstract}
We studied requested urgent Brain CT Scan procedures justification. In addition, we addressed referrer's (physician's) awareness of radiation risks. In doing so, we considered two aspects to the issue. First, we reviewed the records of the requested urgent $\mathrm{CT}$ scan procedures for a sample of 339 patients at Al-Maqassed Hospital, a major hospital in Palestine. Secondly, we surveyed a sample of forty-two referrer's from the same hospital to test their awareness of radiation risks. Our study shows that out of the 339 urgent brain $\mathrm{CT}$ requests, $69.6 \%$ were justified requests and $30.4 \%$ were unjustified. Statistically, these observations implied the following general statement: At least, $25 \%$ of the CT requests at this Hospital are unjustified with a p-value of 0.011 . On the other hand, our survey on referrers shows that $42 \%$ of respondents knew the effective dose of a brain CT scan, $24 \%$ of respondents knew the radiation risks and $14 \%$ of respondents knew about radiation protection. Although the study is limited, yet it shows the need to reduce the number of brain CT examinations and the need to improve their justification. Consequently, the need for regular education and guideline implementation at least in this country is of paramount importance.
\end{abstract}

Keywords: Radiation risk, CT scan, Brain, Ionizing radiation.

\footnotetext{
"Corresponding author: altell.anan@gmail.com.
}

\section{Introduction}

Diagnostic imaging modalities are increasingly applied in health care to diagnose the various injuries and diseases (Merzenich et al., 2012). In spite of the improvements of the medical imaging modalities in the quality of diagnosis and treatment in various medical conditions in both paediatric and adult patients, by using different medical imaging modalities that utilizes ionizing radiation including conventional radiology, fluoroscopy, and Computed Tomography (CT). Scientific studies proved that radiation has biological effects on the organism and it depends on the dose and exposure duration (Squillaro et al., 2018).

The CT has relatively high radiation doses compared with the other ionizing radiation modalities. Thus, the protection against the hazard of radiation is an important issue (IAEA,2009; Remedios, 2011). The principle of radiation protection related to the International Commission on Radiological Protection (ICRP) for ionizing radiation is justification, optimization of protection and application of dose limits (Agency, 2012).

The justification process undergoes the base of risk versus benefit. This base is applied in various techniques, such as ionizing or non-ionizing radiation (Malone et al., 2012). The justification involves using all of the medical team including referring physicians, radiographers, and radiologists (Malone et al., 2012). All examinations in diagnostic radiology shall then be justified to avoid unnecessary irradiation of patients (Remedios, 2011). The number of CT examinations is increasing every year (Brenner et al., 2007, Broder et al., 2006). Referring physicians at the emergency room (ER) who requested radiology examinations in a time-pressured environment need to know how di-

This is an Open Access article distributed under the terms of the Creative Commons Attribution License (http://creativecommons.org/licenses/by/3.0/), which permits unrestricted use, distribution, and reproduction in any medium, provided the original work is properly cited. 
agnostic imaging is indicated as well as the necessity to know the accurate knowledge of the associated risks (Keijzers and Britton, 2010).

By definition of justification, medical exposure is justified when the benefit of the examination is greater than the harm. Previous investigations show that hundreds of examinations requested every year are not justified and most of them are not necessary at all; including the CT examinations((Malone et al., 2012; Teferi et al., 201 ; Hobbs et al.,2018; Vassileva et al., 2012). Also, some of these studies show that there is a prevalent disregard of radiation doses among physicians (Wong et al., 2012; Thomas et al., 2006; Rice et al.,2007; Heyer et al., 2010).

With the increase of radiation exposure towards patients, referring physicians play a greater role in the proper choice of investigation as well as deciding the right choice for the patient. There are some studies that have been conducted on the knowledge about radiation protection for health professionals who work with ionizing radiation (Muhogora et al., 2010). However, they are a few studies that have been applied to the reference of a physician's knowledge regarding the justification of the procedures (Moifo et al., 2018).

The goal of this study is to determine whether previous CT examinations done at Makassed Hospital in Jerusalem on the patients who entered the emergency department were justified. To accomplish this, patient files of 339 urgent brain CT examinations were retrospectively reviewed. Also, a questionnaire made up of 42 physicians was created to analyse the referring physician's awareness of radiation risks and weather the requests of medical imaging procedures can be justified or not.

\section{Materials and Methods}

\section{A Sample of Patients}

Participants chosen for the study were patients who urgently referred from the ER for 6 months from the first of January to the 30th of June 2018. Approximately 339 patients with suspected or confirmed head injury with or without other major trauma were studied. The study referred to the archive of the hospital through the hospital information system (HIS) which records all observations before examinations are done. Then, the results of those examinations are written and then compared.

An institutional review approval was obtained for this prospective study. The patient consent was conceded, but the patient particularity was conserved by using the patient ID without mentioning the name of the patient. Emergency department patients were included, other departments were excluded. Also, emergency department patients who during the morning working shift 7:00 AM to 3:00PM as elective and scheduled were excluded while patients between 3:00 pm to 7:00 am were included.

The data collection method included reviewing patient notes during emergency situations. We used the HIS archive which is used in the hospital. The data for each patient included age, gender, clinical data (obtained from the ER physician), the exam indications and the findings written by radiologists. The patients who have no written finding reports were also excluded.

\section{A Sample of Doctors}

Fourty two (42) referring physicians from the hospital believe that they can justify at least ten brain CT requests of image procedures per week. Thus, a plan has been developed to interview each potential respondent in the hospital. Each meeting session concluded explanations that were provided from the respondent according to the objectives of the survey, the confidentiality of the responses and the anonymous nature of the survey model. Once approved, the pre-screened questionnaire is administered to the respondent. Sampling was continuous and easy going. Radiologists and radiographers were excluded. The questionnaire consisted of a section on the person interviewed (gender, qualification, years of experience), a section on knowledge and the exercise of justification for the examination of the required medical radiation with emphasis on the effective dose of brain CT scans and radiation risks. Other questions focused on the responsibility of justification and the daily practice of referring physicians on the justification of the required examinations and the principles of radiation protection. The main part of the questionnaire is shown in Appendix 1.

\section{Data Analysis}

Two experts in radiology with the new south Wales Ministry of Health NSW Health clinical practice guideline (Haydon, 2013) wrote the following findings for each exam. It also assessed the justification of the performed examinations based on the referrals. The analyses of examinations were divided into two groups; justified and unjustified, and the percentage of the two groups were calculated. The guideline requirements for indicating the potentially significant mild head injury summarized in Table 1.

This scale can be applied with the time it took to deal with the patients. However, because our work is on the retrospective study; this scale cannot be applied. Thus, researchers put a scale

Table 1. Initial management of adult closed head injury (NSW Health algorithm).

\begin{tabular}{ll}
\hline A-WPTAS, Abbreviated Westmead PTA Scale; GCS, Glasgow Coma Scale. \\
\hline 1 & GCS $<15$ at 2 h after injury. \\
2 & Deterioration in GCS \\
3 & Focal neurological deficit \\
4 & Clinical suspicion of skull fracture \\
5 & Vomiting (especially if recurrent) \\
6 & Known coagulopathy or bleeding disorder \\
7 & Age $>65$ years \\
8 & Post-traumatic seizure \\
9 & Prolonged loss of consciousness ( $>5$ min) \\
10 & Persistent post-traumatic amnesia (A-WPTAS $<18 / 18$ at 4 h after injury \\
11 & Persistent abnormal alertness/behavior/cognition \\
12 & Persistent severe headache \\
13 & Large scalp hematoma or laceration \\
14 & Multi-sy stem trauma \\
15 & Dangerous mechanism \\
16 & Known neurosurgery/neurological impairment \\
17 & Delayed presentation or representation \\
\hline
\end{tabular}


that consists of four groups from 1 to 4 as follows:

Group 1 (G1): Brain CT examination of cases related to head trauma and its involvement with road traffic accidents (RTA), motor vehicle accidents (MVA) and falling down cases.

Group 2 (G2): Brain CT examinations of patients with vomiting, dizziness, seizure, and convulsion.

Group 3 (G3): Brain CT examinations of patients suffering loss of consciousness, sleepiness, side body weakness, numbness, and severe headache.

Group 4 (G4): Brain CT examinations after surgery (postoperative).

The statistical analysis was obtained using the Statistical Package for Social Sciences.

\section{Results}

As we mentioned, a total of 339 urgent brain $\mathrm{CT}$ request forms were received during the study period ( 6 months). Of these; $209(61.65 \%)$ were males and $130(38.35 \%)$ were females. $111(32.74 \%)$ were children less than 16 years, 228 $(67.26 \%)$ were over 16 . Out of the total 339 CT request forms, 105 (30.98\%) received were for patients in the ER who stayed in different departments inside the hospital, while 234 $(69.02 \%)$ brain CT requests were for patients who were at the ER and who stayed under observation for 24 hours and were then discharged the next day. Out of the 22 patients who were excluded from the study; 10 of them were not given a report due to their impatience. The remaining patients did not have an examination request. Table 2 and Figure 1 provide summary of the distribution of age, gender, and patients who were included as well as those were excluded in the study.

As it is a retrospective study, it is difficult to justify every request based on clinical data only. In our study, we consider the exam retrospectively justified, if the note demonstrate that the patient belongs to one of the four group. Table 3 and figure 2 show the distribution of the justified and non-justified requests.

The study estimates that $236(69.6 \%)$ requests of the total 339 was retrospectively considered a justified request, as the request fall in one of the four group, while 103 (30.4\%) was not fulfilling the justification criteria applied to our study.

Statistically: The sample size is $n=339$, the number of unjustified requests is 103 .

Let $\mathrm{P}$ denotes the proportion of the unjustified requests at this Hospital in general, and $\mathrm{P}^{v}$ denotes the proportion of the unjustified requests in our sample study. Then, from the test Ho: $\mathrm{P} \leq 0.25$ and Ha: $\mathrm{P}>0.25$, we have the following: a $95 \%$ confidence interval for $\mathrm{P}$ is $0.255<\mathrm{P}<0.353$, and a $\mathrm{p}$-val$\mathrm{ue}=0.011$ as shown in Figure 3. This strongly support the conclusion that $\mathrm{P}>25 \%$ (Figure 3 ).

Our two experts wrote the difference between the clinical data (indication) with the data findings (reports). Out of the 339 patients, $23(6.78 \%)$ had no radiology report, while 104 (30.67\%) had normal results after the brain CT examination.

This study estimates that 26 patients $(7.66 \%)$ had mismatches between the indications and the correct findings after the examinations were done. Keeping in mind, a brain CT indication is not a hard requirement to achieve from ER physicians. 129 $(38.05 \%)$ out of the 339 patients had matchings between the final reports with the clinical data, while $57(16.81 \%)$ patients had mismatches between the final reports and exam indications. However, it's still important to request brain exams from ER physicians because the reports do show emergency findings inside these cases.

In essence, to check the accuracy for brain CT examination requests from ER physicians, while excluding radiologist re-

Table 2. Demographics of clinical study cohort.

\begin{tabular}{lccccccc}
\hline Demographics & January & February & March & April & May & June & Total \\
\hline Male split & 26 & 28 & 26 & 42 & 40 & 47 & 209 \\
Female split & 15 & 13 & 23 & 28 & 29 & 22 & 130 \\
Children patients $<$ 16 & 8 & 7 & 10 & 38 & 25 & 23 & 111 \\
another patient $>\mathbf{1 6}$ & 33 & 34 & 39 & 32 & 44 & 46 & 228 \\
Patients included & 36 & 40 & 49 & 67 & 64 & 61 & 317 \\
Patients Excluded & 5 & 1 & 0 & 3 & 5 & 8 & 22 \\
Inpatient & 15 & 12 & 23 & 22 & 15 & 18 & 105 \\
Outpatient & 26 & 29 & 26 & 48 & 54 & 51 & 234 \\
\hline
\end{tabular}

Table 3. Documentation for justified and non-justified requests.

\begin{tabular}{|c|c|c|c|c|c|c|c|c|}
\hline \multicolumn{2}{|c|}{ Month } & \multicolumn{4}{|c|}{$\mathbf{J}$} & \multirow[t]{2}{*}{ N.J } & \multirow[t]{2}{*}{$\% \mathrm{~J}$} & \multirow[t]{2}{*}{ \%N.J } \\
\hline & $\mathbf{T}$ & $G_{1}$ & $G_{2}$ & $G_{3}$ & $G_{4}$ & & & \\
\hline January & 41 & 5 & 11 & 2 & 6 & 17 & 58.53 & 41.46 \\
\hline February & 41 & 9 & 3 & 14 & 3 & 12 & 70.74 & 29.26 \\
\hline March & 49 & 10 & 3 & 15 & 10 & 11 & 77.56 & 22.44 \\
\hline April & 70 & 22 & 6 & 15 & 7 & 20 & 71.43 & 28.57 \\
\hline May & 69 & 23 & 4 & 6 & 10 & 26 & 62.32 & 37.68 \\
\hline June & 69 & 29 & 9 & 8 & 6 & 17 & 75.37 & 24.63 \\
\hline Total & 339 & 98 & 36 & 60 & 42 & 103 & 69.6 & 30.4 \\
\hline
\end{tabular}




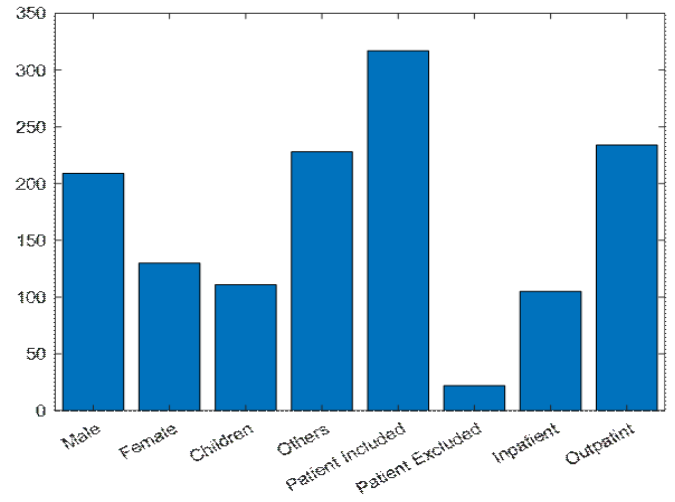

Figure 1. Distribution of age, gender, and patients who were included as well as those were excluded in the study.

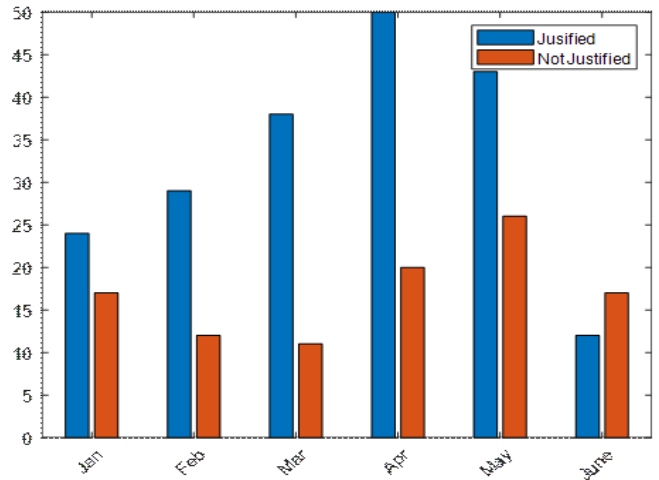

Figure 2. Distribution of the justified and non-justified requests during the period of study.

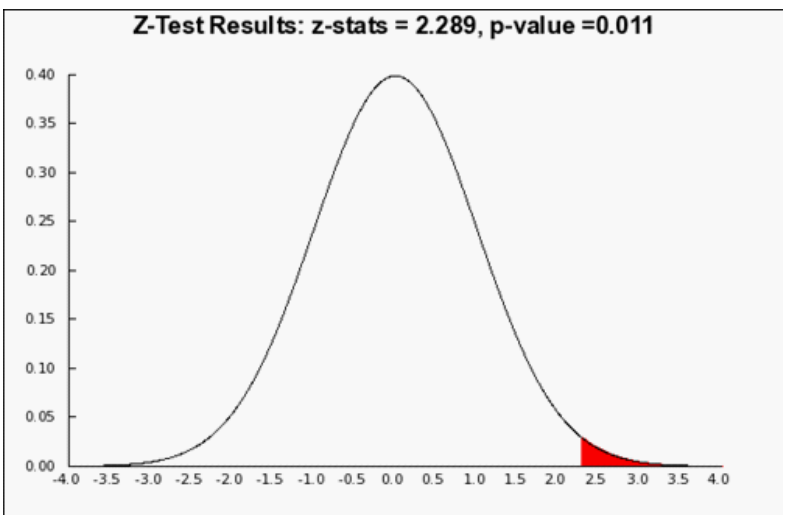

Figure 3. Test on the proportion of the unjustified requests.

ports, we distributed three categories of findings:

a. True-negative: acts for the patients, who had a request to do an examination with a result. This category consists of both patients with matchings between the examination indication and the final report and those patients who have a mismatching between the clinical data with the radiology report. According to the reports, it was estimated that $54.86 \%$ show an importance for the exam to be made.
Table 4. The accuracy of ED physicians for order the brain CT exams.

\begin{tabular}{lccccc}
\hline & No & Normal & Matching Report & \multicolumn{2}{c}{ Mismatching } \\
Report & Report & with Clinical Data & T & F \\
\hline January & 3 & 8 & 18 & 10 & 2 \\
February & 1 & 10 & 14 & 7 & 9 \\
March & 4 & 11 & 25 & 8 & 1 \\
April & 3 & 26 & 23 & 12 & 6 \\
May & 5 & 19 & 26 & 15 & 4 \\
June & 7 & 30 & 23 & 5 & 4 \\
Total & $\mathbf{2 3}$ & $\mathbf{1 0 4}$ & $\mathbf{1 2 9}$ & $\mathbf{5 7}$ & $\mathbf{2 6}$ \\
\hline
\end{tabular}

Table 5. The questionnaire results.

\begin{tabular}{ccc}
\hline Questions & Correct Answer & Correct Answer Percent \\
\hline Q1 & C & $42 \%$ \\
Q2 & C & $3 \%$ \\
Q3 & D & $24 \%$ \\
Q4 & D & $14 \%$ \\
Q5 & A & $23 \%$ \\
\hline
\end{tabular}

b. True-positive: refers to the patients, an estimation of $30.67 \%$ who had a request to do the examination with normal results. c. The third category: consists of patients who had a request to do the examination, but the examination did not undergo the emergency exam, with an estimated $7.66 \%$. From these results, we come to know that the accuracy of ER doctors only reached $54.86 \%$. Table 4 summarized these results. There were 130 patients $(38.34 \%)$ that received a brain $\mathrm{CT}$ without having the documented criteria required to justify the investigation based on the NSW Health program.

According to the questionnaire on doctors, the results for each question are shown in Table 5.

According to this survey, 18 referrers (42\%) of the 42 questioned referrers know the effective dose of the brain CT scan. However, just 13 referrers (3\%) has known that the effective dose of one brain CT scan is equal to the effective dose of 100 chest $\mathrm{x}$-ray (a routine emergency radiological exam).

In despite the that all referrers believe ionizing radiation is a risk factor for cancer development, only $24 \%$ of the them have known the exact excess risk of cancer from radiation.

Six referrers $(14 \%)$ of all respondents knew the basic principles of radiation protection, and 10 referrers $(23 \%)$ consider a local or an international protocol when request an urgent brain CT scans.

\section{Discussion}

In this current study, the requests for brain $\mathrm{CT}$ examinations over a six month period are reviewed. Approximately 30.4\% was not justified, the degree of justification was only about $69.6 \%$. Our present study shows that the un-justification of brain CT examinations was statistically significant. For the children, the un-justification exams are at a significant high, with the total number of examinations for children being less than adult patients. This result is similar to a previous study done in Germany (Merzenich et al., 2012). The number of brain CTs carried out on adult patients increased significantly, which were ordered after 15:00 o'clock comparing with the time before. 
Despite the recommendations, radiologists were not regularly consulted before brain CT's were requested for adult patients. The analysis of justification for a brain $\mathrm{CT}$ is also challenging because of the multiple and variable indications.

According to the questionnaire on a sample of referring physicians, the level of knowledge of referring physicians regarding justification for the request of radiating examinations was not satisfactory. This is harmonious with many previous studies: o'Sullivan et al which shows that $87 \%$ of the study population and $89 \%$ of controls considered that they had never been exposed to lectures or teaching focussed on radiation protection; Soye and Paterson which demonstrated that Fiftyfour percent of students reported a moderate confidence in their knowledge about radiation and risks. Borgen et.al study shows that the mean radiation knowledge score was 30.4/71. Most respondents underestimated doses from high-dose imaging, e.g., barium enema (94.7\%), chest CT $(57.7 \%)$ and abdominal CT (52.7\%). Limited radiation knowledge was not compensated by using guidelines. Only $20 \%$ of physicians and $72 \%$ of non-physicians used referral guidelines and the Krille.et al systemic review which showed moderate to low knowledge among physicians concerning radiation doses and the involved health risks (O'Sullivan, 2010; Soye and Paterson, 2000; Zewdneh et al., 2012; Borgen, 2010; Krille,2010). This level of knowledge has not changed with professional experience or qualifications. The explanation might be the lack of initial training during medical studies and the absence of regular structured education in Hospitals on radiation protection.

There are several limitations to this study. The major limitation is that this study contains information from one institution only. The patient number selected for the justification evaluation is also low. The study dealt with brain CT examinations only. The data collection required to deal with the patients at the time of an examination to determine which patients fitted the criteria. Also, the study concerned the ER and not all hospital departments and it only focused on the patients after the 15:00 o'clock.

In general, it is probably impossible to reach $100 \%$ justification. However, it is still essential to develop a justification process. Regular updating of referral guidelines with the computer system used at the hospital to increase justification. Continuous and easy communication system between referrers, radiographers and radiologists will improve the unjustifiable exam by feedback for each request. PACKs and HIS system make these communications easier and smooth.

In conclusion, the study has shown that it is possible to reduce the number of brain $\mathrm{CT}$ examinations and to improve their justification by regular education, guideline implementation and to educate physicians about the ionizing radiations related to medical imaging, given their legal responsibility as dispensers under the ionizing radiation regulations (medical exposure) in the hospital.

\section{References}

Agency HP (2012) Annals of the ICRP. 1 to 2005.

Borgen L, E Stranden, and A Espeland (2010) Clinicians' justification of imaging : Do radiation issues play a role ? Insights Imagins 1 (3): 193-200. http://doi.org/10.1007/s13244-010-0029-4.

Brenner DJ and EJ Hall (2007) Computed Tomography — An Increasing Source of Radiation Exposure. NEJM 357 (22): 2277-2284.

Broder J and DM Warshauer, (2006) Increasing utilization of computed tomography in the adult emergency department, $2000-2005$. Emergency Radiology 13 (1): 25-30.

Haydon NB (2013) Head injury: Audit of a clinical guideline to justify head CT. J. Med. Imaging Radiat. Oncol. 57 (2): 161-168. https:// doi.org/10.1111/1754-9485.12007.

Heyer CM, J Hansmann, SA Peters, and SP Lemburg (2010) Paediatrician awareness of radiation dose and inherent risks in chest imaging studies - A questionnaire study. Eur. J. Radiol. 76 (2): 288-293 http://doi.org/10.1016/j.ejrad.2009.06.014 .

Hobbs JB, N Goldstein, KE Lind, D Elder, GD Dodd, and JP Borgstede (2018) Physician Knowledge of Radiation Exposure and Risk in Medical Imaging. J. Am. Coll. Radiol 15 (1 Pt A): 34-43, http://doi. org/10.1016/j.jacr.2017.08.034 .

International Atomic Energy Agency (IAEA) (2009) Report of a consultation on justification of patient exposures in medical imaging. Radiat. Prot. Dosimetry 135 (2): 137-144.

Keijzers GB and CJ Britton (2010) Doctors' knowledge of patient radiation exposure from diagnostic imaging requested in the emergency department. MJA 193 (8): 450-453. http://doi. org/10.5694/j.1326-5377.2010.tb03998.x.

Krille L, G Hammer, H Merzenich, and H Zeeb (2010) Systematic review on physician's knowledge about radiation doses and radiation risks of computed tomography. Eur. J. Radiol 76 (1): 36-41. https:// doi.org/10.1016/j.ejrad.2010.08.025.

Malone J, R Guleria, C Craven, P Horton, H Järvinen, J Mayo, G O'reilly, E Picano, D Remedios, J Le Heron, M Rehani, O Holmberg, and R Czarwinski (2012) Justification of diagnostic medical exposures: Some practical issues. Report of an International Atomic Energy Agency Consultation", BJR 85 (1013): 523-538. https:// www.birpublications.org/doi/10.1259/bjr/42893576.

Merzenich H, L Krille, G Hammer, M Kaiser, S Yamashita, and H. Zeeb (2012) Paediatric CT scan usage and referrals of children to computed tomography in Germany-a cross-sectional survey of medical practice and awareness of radiation-related health risks among physicians. BMC Health Serv. 12 (1): 1-7.

Moifo B, AL Edzimbi, H Tebere, J Tambe, RN Samba, and JG Fotsin (2014) Referring Physicians' Knowledge on Justification of Medical Exposure in Diagnostic Imaging in a Sub-Saharan African Country, Cameroon. Open J. Radiol 4: 60-68. http://doi.org/10.4236/ ojrad.2014.41008.

Muhogora WE, NA Ahmed, JS Alsuwaidi, A Beganovic, O CirajBjelac, V Gershan, E Gershkevitsh, E Grupetta , MH Kharita, N Manatrakul, B Maroufi, M Milakovic, K Ohno, L Ben Omrane, J Ptacek, C Schandorf, Shaaban MS, N Toutaoui, D Sakkas, JS Wambani, MM Rehani (2010) Paediatric CT examinations in 19 developing countries: Frequency and radiation dose. Radiat Prot Dosimetry 140 (1): 49-58. http://doi.org/10.1093/rpd/ncq015.

O'Sullivan J, OJ O'Connor, K O'Regan, B Clarke, LN Burqouyne, MF Ryan, MM Maher (2010) An assessment of medical students' awareness of radiation exposures associated with diagnostic imaging investigations," Insights imaging 1 (2): 86-92.

Remedios D (2011) Justification: How to get referring physicians involved',Radiation protection dosimetry 147 (1-2): 47-51 . https:// doi.org/10.1093/rpd/ncr263.

Rice HE, DP Frush, MJ Harker, D Farmer, and JH Waldhausen,(2007) Peer assessment of pediatric surgeons for potential risks of radiation exposure from computed tomography scans. J. Pediatr. Surg, 
42 (7): 1157-64.

Soye JA and A Paterson (2008) A survey of awareness of radiation dose among health professionals in Northern Ireland. BJR 81 (969): 725-729 http://doi.org/10.1259/bjr/94101717.

Squillaro T, G Galano, R De Rosa, G Peluso, U Galderisi (2018) Concise Review: The Effect of Low - Dose Ionizing Radiation on Stem Cell Biology : A Contribution to Radiation Risk. Stem Cells 36: 1149- 1153.

Teferi S, D Zewdeneh, and S Bekele (2018) Pediatric Residents ' and Medical Interns' Awareness about Pediatric Ionizing Radiation Dose from Computed Tomography and Its Associated Risks in Tertiary Hospital in Ethiopia. Ethiopian Journal of Health Science 28 (4): 383-392.

Thomas KE, JE Parnell-Parmley, S Haidar, R Moineddin, E Charkot, GB David, C Krajewski (2006) Assessment of radiation dose awareness among paediatricians. Paediatric Radiology 36 (8): 82332.

Vassileva J, MM Rehani, H Al-Dhuhli, HM Al-Naemi, JS AlSuwaidi,K Appelgate, D Arandjic, EH Bashier,A Beganovic, T Benavente, T Bieganski, S Dias, L El-Nachef, D Faj, ME GamarraSánchez, J Garcia-Aguilar, L Gbelcová, V Gershan, E Gershkevitsh, E Gruppetta, A Hustuc, S Ivanovic, A Jauhari, MH Kharita, S
Kharuzhyk, N Khelassi-Toutaoui, HR Khosravi, H Khoury, D Kostova-Lefterova, I Kralik,L Liu, J Mazuoliene, P Mora,W Muhogora, P Muthuvelu,L Novak, AS Pallewatte, M Shaaban,E Shelly, K Stepanyan, EL Teo, N Thelsy, P Visrutaratna, A Zaman, and D Zontar (2012) IAEA survey of pediatric CT practice in 40 countries in Asia, Europe, Latin America, and Africa: Part 1, frequency and appropriateness. Am. J. Roentgenol. 198 (5): 1021-1031. http://doi. org/10.2214/AJR.11.7273 .

Wong CS, B Haung, HK Sin, WL Wong, KL Yiu, and T Chu Yiu Ching (2012) A questionnaire study assessing local physicians, radiologists, and interns' knowledge and practice pertaining to radiation exposure related to radiological imaging. Eur. J. Radiol., 81 (3): e264-8. http://doi.org/10.1016/j.ejrad.2011.02.022.

Yates D, R Aktar, and J Hill (2007) Assessment, investigation, and early management of head injury: Summary of NICE guidance. BMJ 335 (7622): 719-720. http://doi.org/10.1136/bmj.39331.702951.47.

Zewdneh D, ST Dellie, and T Ayele, (2012) A Study of Knowledge \& Awareness of Medical Doctors Towards Radiation Exposure Risk At Tikur Anbessa Specialized Referral. IOSR Journal of Pharmacy and Biological Sciences 2 (4): 1-5. http://doi.org/10.9790/30080240105 .

\section{Appendix 1.}

Survey questionnaire with correct answers.

Q1: What is the effective dose of brain CT scan:

$$
\begin{aligned}
& 0.1 \mathrm{msv} \\
& 1 \mathrm{mSv} . \\
& 2 \mathrm{mSv} . \\
& 4 \mathrm{mSv} . \\
& 10 \mathrm{mSv} \text {. }
\end{aligned}
$$

Q2: if we consider the chest $\mathrm{x}$-ray effective dose as 1 unit, the effective dose of the brain CT scan will be

\section{1.}

10.

100.

1000

10000

Q3: the excess risk of cancer from radiation is

\section{$1 / 10000 \mathrm{mSv}$.}

$1 / 100000 \mathrm{mSv}$.

$1 / 100 \mathrm{mSv}$.

$1 / 4000 \mathrm{mSV}$.

No increase in cancer risk.
Q4: The radiation protection philosophy that promotes the use of the least amount of radiation possible for medical imaging is termed:

\section{NCRP \\ NRC \\ ICRP \\ ALARA}

Q5: When you order a brain CT scan especially in trauma patients, do you consider any local or international guidelines.

Yes.

No. 